gression line of mass on volume : from this line the mass can be found by measurements of volume under similar conditions of distributions of errors and true values to those in the preliminary sample. The of errors and true values to those in the preliminary sample. The
regression line of mass on volume provides the best estimate of true mass from observed volume. The use of the regression line to estimate mass from observed volume. The use of the regression line to estimate density is only valid if measurements of volume are free from error : the use of the line of best flt to estimate mass from vill, in general, lead to biased estimates.

from mass will, in general, lead to biased estimates. nected by a linear relation then the regression of measured value of nected by a linear relation then the regression of measured value of one variable (the dependent variable) on the measured value of the possible to prove that under this condition the necessary and sufficient possible to prove that under this condition the necessary and sufficient condition for linearity is that the cumulant generating function of muitiple of the cumulant generating function of the distribution of true values of the independent variable.

The estimate of Kummell's involves a knowledge of the ratio of the standard deviations $\beta$ : Austen and Pelzer have suggested that, if $\beta$ is unknown, one should assume $\beta=\rho$, the slope of the line, leading to the estimate

$$
p=\left\{\Sigma w^{2} / \Sigma v^{2}\right\}^{1 / 2} .
$$

It should be noticed that this estimate is, in general, inconsistent, that is, as the sample size increases indefinitely the estimate, instead of tending to the true slope, tends to another value. It is the minimum requirement of any estimate that it be consistent, and hence the use of (1) is not satisfactory; it has previously been proposed by Dent ${ }^{6}$. of (1) is not satisfactory ; it has previously been proposed by Dent ${ }^{6}$. a knowledge of $\beta$, and if the distribution of errors and true values are a knowledge of $\beta$, and if the distribution of errors and tr

normal, then no cons solution
Seares's

$$
\rho=\left\{\frac{\bar{w}^{2}-\sigma_{v v}^{2}}{\bar{v}^{2}-\sigma_{v}^{2}}\right\}^{1 / 2}
$$

requires a knowledge of $\sigma_{w}, \sigma_{v}$ and not merely their ratio, so that as it assumes more known, 1 should have expected it to have given a better estimate of $\rho$ than Kummell's.

It is hoped to publish a fuller discussion of these points elsewhere, together with a proof of the above theorem and some generalizations of it: the work has been carried out as part of the research programme of the National Physical Laboratory, and this letter is published by permission of the Director.

National Physical Laboratory,

D. V. LINDLET

Teddington, Middlesex.

$$
\text { June } 24 .
$$

${ }^{1}$ Nature, 157, 693 (1946).

'Analyst (Des Moines), 6, 97 (1879)

"Statistical Adjustment of Data" (Wiley, 1944), 184.

'Metron, 13, 3 (1937).

Ann. Math. Stat., 11, 284 (1940).

- Ann. Math. Stat., 11, $284(1940)$.

\section{Elimination of Certain Divergencies in Quantum Electrodynamics}

THE ' $\lambda$-limiting process', given by Wentzel and improved by Dirac, gives a flnite classical theory for the interaction of a point-charge with its own field. A new mathematical method due to $\mathbf{M}$. Riesz of solving the wave equation by means of analytleal continuation gives the same finite equation of motion for a point-charge as this '-limiting process'.

In previous papers we have applied these mathematical methods to quantum electrodynamies and to meson theory, solving the ordinary and the meson wave equation respectively by analytical continuation. In the following we will generalize these calculations.

We consider the interaction of an electron fleld $\psi$ satisfying Dirac's equation, and an electromagnetic field $A$. When second quantization is used, $\psi$, too, becomes a matrix wave function. We work with the
Heisenberg picture, with matrices varying with $t$ and the state vector constant. The equations are

$$
\begin{gathered}
{\left[\frac{i \hbar}{c} \frac{\partial}{\partial t}-i \hbar \alpha^{i} \frac{\partial}{\partial x^{i}}-\beta m c\right] \psi=\frac{e}{c} A_{\mu} \alpha^{\mu} \psi .} \\
\square A_{\mu}=4 \pi e \psi^{*} \alpha_{\mu} \psi .
\end{gathered}
$$

We assume the usual commutation relations. The self-energy terms are, in the second approximation,

$$
\begin{gathered}
W=W_{1}+W_{2}=\frac{e^{2}}{2} \iiint d V\left[\psi^{0 *} \psi^{0} A_{0}^{1}-\frac{1}{c} \psi^{0 *} \vec{\alpha} \psi^{0} \overrightarrow{A^{1}}\right] \\
-\frac{e^{2}}{2 c} \iiint\left[\psi^{* *} \vec{\alpha} \psi^{0}+\psi^{0 *} \vec{\alpha} \psi^{1}\right] \vec{A}^{0} .
\end{gathered}
$$

We ${ }^{3}$ have obtained $A^{1}$ by analytical continuation with respect to a warameter $\alpha$ for a certain value $\alpha=2$. Physical expressions containing parameter $\alpha$ for a certain value $\alpha=2$. Physical expressions containing
$A^{1}$ can be defined as functions of $\alpha$ and calculated by analytical con$A^{1}$ can be defined as functions of $a$ and calculated by analytical conformerly divergent expressions become finite (for example, $W_{\mathrm{i}}=0$ ), but other divergencies still remain, for example, the second selfenergy term $W_{2}$. Introducing in addition to the " $\lambda$-limiting process" the hypothesis of positive- and ne

Hitherto, we have solved the wave equation by analytical con-
tinuation and the Dirac equation in the ordinary way The Dirac equation is also, however, a hyperbolic equation, the solution of which, and thereby the corresponding energy expressions, can be given by and thereby the corresponding energy expressions, can be given by has no counterpart in classical theory.

Introducing

$x^{0}=c t, e^{0}=\beta, e^{i}=-\beta \alpha^{i}, \nabla=\Sigma e^{v} \frac{\partial}{\partial x^{v}}, \mu=m c / \hbar$.

we write the Dirac equation

$$
(\nabla+i \mu) \psi=-\frac{i e}{\hbar c} A_{\mu} \beta \alpha^{\mu} \psi \text {. }
$$
As $\nabla^{2}=\square$, we have $(\nabla-i \mu)(\nabla+i \mu)=\square+\mu^{2}$
The solution of $\left(\square+\mu^{2}\right) \psi=f(P)$ is the analytical continuation to $\alpha=2$ of

$$
\begin{array}{r}
V^{\alpha} f(P)=\frac{1}{2^{\alpha / 2} 2 \pi \Gamma\left(\frac{\alpha}{2}\right)} \iiint_{D_{S}^{P}} f(Q)\left(\frac{r_{P Q}}{\mu}\right)^{\frac{\alpha-4}{2}} \\
\quad J_{\frac{\alpha-4}{2}\left(\mu r_{P Q}\right) d Q .} .
\end{array}
$$

where $r P Q$ is the Lorentz distance between $P$ and $Q$, and $D_{g}^{P}$ is the four-dimensional domain bounded by the retrograde light-cone with its top in $P$ and by the space $S$. Riesz ${ }^{2}$ has obtained the $\alpha$ function corresponding to the operator $\nabla$. We now seek the functions $L_{1}{ }^{a}$ and $L_{2} \alpha$ corresponding to the operators $\nabla+i \mu$ and $\nabla-i \mu$ respectively. We find

$$
\begin{aligned}
L_{1} \alpha f(P)=\exp \left(\frac{i \pi \alpha}{2}\right)\left[\cos \frac{\pi \alpha}{2} V \alpha f(P)-\right. & \\
& \quad i \sin \frac{\pi \alpha}{2}\left(\nabla-i(\nu) V^{\alpha+1} f(P)\right],
\end{aligned}
$$

$L_{\mathrm{s}} \alpha_{f(P)}$ is obtained from $L_{1} \alpha_{f(P)}$ by sukstituting $\nabla+i \mu$ for $\nabla-i \mu$. As $(\nabla+i \mu) L_{1} a_{f}(P)=L_{2} a^{-1} f(P)$ and $L_{2}{ }^{0} f(P)=f(P)$, the solution of the Dirac equation is obtained by analytical continuation to $a=1$. by analytical continuation. Inserting

$$
\psi^{1 \alpha}=(\nabla-i \mu) V^{\alpha}+1\left[\left(-\frac{i}{\hbar c}\right) A_{\mu}^{0} \beta \alpha \mu \psi^{0}\right]+\text { (terms }
$$

vanishing for $\alpha=1$ ) for $\psi^{1}$ in $W_{2}$, we get an expression $W_{2} \alpha$. Owing to $\boldsymbol{A}^{\mathbf{0}}$, the matrix wave function for the electromagnetic field in empty space. we get sums over all photons. In the one-electron: case. $\psi^{0}$ contains only one term which is not zero. We perform the operation $\nabla-i \mu$. For an electron at rest the terms containing $\beta \alpha^{i} \frac{\partial}{\partial x^{i}}$ are zero. After further calculation we get for the other terms, when neglecting the retardation, an integrand which contains a sum over all photons

$$
\sum_{\lambda} \exp \left(\frac{i}{\hbar} k_{\lambda}\left(X_{Q}-X_{P}\right)\right)=\delta\left(X_{Q}-X_{P}\right) \text {. }
$$

A calculation analogous to that for $W_{1}^{\alpha}$ gives ${ }^{4,5}$ for $W_{2}{ }^{\alpha}$ an expression convergent for $2<\alpha<4$, which can be continued analytically to find the analytical continuation to $\alpha=1$. We find $W_{2}=-\frac{e^{2} \mu}{8}$. Thus solving both the wave equation and the Dirac equation by
analytical continuation, we find without further hypotheses fnite expressions for the second approximation of the interaction energy. A fuller account of this work will be presented in the Arkiv. $f$. Mat. Astr. o. Fys., Stockholm.

Torsten GUsTaFsox University of Lund. June 15 .

${ }^{1}$ Riesz, M., Congrès Internat. Math. Oslo, 2, 44 (1936). 2 Riesz, M., Confér. à la Réunion internat. d. Math. à Paris, 1937 (1939).

3 Gustafson, T., Lunds Fysiogr. S. Förh., 15, 28 (1945).

- Gustafson, T., Lunds Fysiogr. S. Forh., 16, 2 (1946).

${ }^{5}$ Gustafson, T., Nature, 157, 734 (1946).

\section{Practical Control of Wireworm with 'Gammexane'}

IN Nature of June 8, Messrs. Golightly and Hogg refer to our statement in an earlier issue that wireworm populations had been reduced by as much as 65 per cent following the application of 'Gammexane'. In view of a misconception that has evidently arisen, we would state that this 65 per cent measured the difference between the populations of treated and untreated plots when sampled at the same time, and had no connexion with the well-known natural fall in population that occurs connexion with the

We realize that the spectacular effect of 'Gammexane' treatment may not be due solely to reduction of the wireworm population, and also that other pests may sometimes be affected by these applications. It is hoped to publish a full account of our trials shortly

Jealott's Hill Research Station, . J. ThOMA

Jealott's Hill Resea H. R. JAMESON Berks. 